\title{
Identification and quantitation of amylase trypsin inhibitors across cultivars representing the diversity of bread wheat
}

Utpal Bose ${ }^{1}$, Angéla Juhász ${ }^{2}$, James A. Broadbent ${ }^{1}$, Keren Byrne ${ }^{1}$, Crispin A. Howitt ${ }^{2}$, Michelle L. Colgrave ${ }^{1^{*}}$

${ }^{1}$ CSIRO Agriculture and Food, 306 Carmody Rd, St Lucia QLD 4067, Australia; ${ }^{2}$ CSIRO Agriculture and Food, GPO Box 1700, Canberra ACT 2601, Australia;

*Corresponding author: Michelle L. Colgrave, CSIRO Agriculture and Food, 306 Carmody Rd, St Lucia QLD 4067, Australia; Phone: +61 73214 2697; Fax: +61 73214 2900; Email: michelle.colgrave@csiro.au

Table of contents:

Figure S1: Multiple sequence alignment showing the conservation of cysteines $(\geq 50 \%)$ in ATIs. Identified protein sequences were aligned with ATIs identified in the bread wheat reference genome.

Table S1: Wheat cultivars and MAGIC parent lines used in the present study.

Table S2: Homology search (BLASTp analysis) of ATI peptides from wheat cultivars monitored by sMRM experiment. Peptides marked as (*) asterisk are plotted in Figure 3 and 5. 


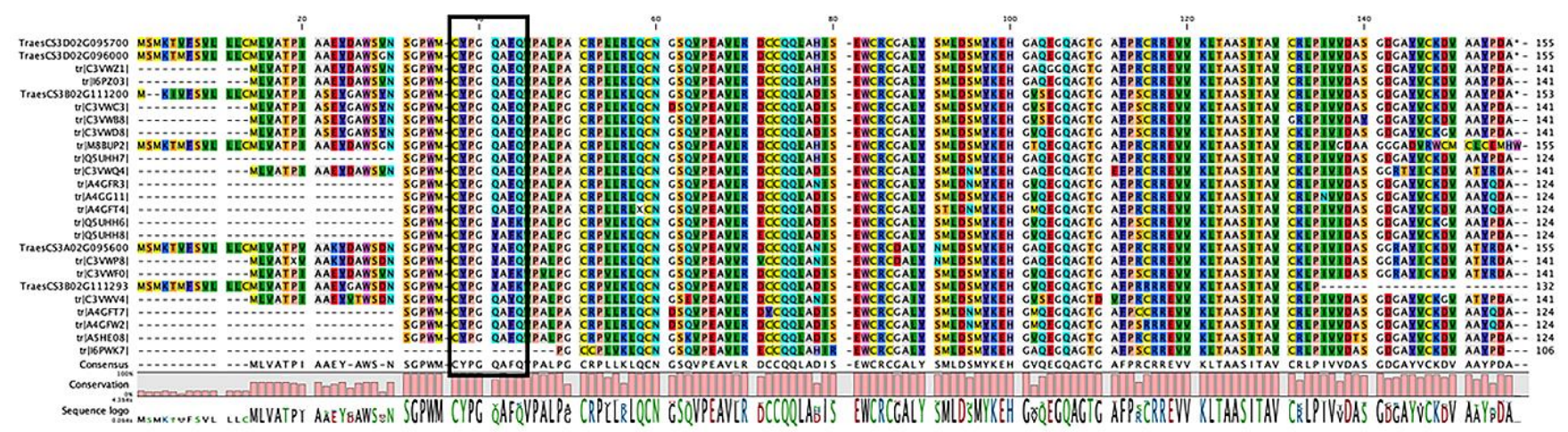

Figure S1: Multiple sequence alignment showing the conservation of cysteines $(\geq 50 \%)$ in ATIs. Identified protein sequences were aligned with ATIs identified in the bread wheat reference genome.

Table S1: Wheat cultivars and MAGIC parent lines used in the present study.

\begin{tabular}{|l|l|}
\hline \multicolumn{2}{|c|}{ Commercial wheat cultivars } \\
\hline$\#$ & Name \\
\hline C1 & Janz \\
\hline C2 & Sunvale \\
\hline C3 & Diamond Bird \\
\hline C4 & Calingiri \\
\hline C5 & Wyalkatchem \\
\hline C6 & EGA Wedgetail \\
\hline C7 & Ventura \\
\hline C8 & EGA Gregory \\
\hline C9 & EGA Bonnie Rock \\
\hline C10 & Yitpi \\
\hline C11 & EGA Kidman \\
\hline C12 & Magenta \\
\hline C13 & Mace \\
\hline C14 & Longreach Scout \\
\hline C15 & Chara \\
\hline
\end{tabular}

\begin{tabular}{|l|l|}
\hline \multicolumn{2}{|c|}{ MAGIC parent lines } \\
\hline M1 & Alsen \\
\hline M2 & AC Barrie \\
\hline M3 & Baxter \\
\hline M4 & Pastor \\
\hline M5 & Westonia \\
\hline M6 & Volcani \\
\hline M7 & Xiaoyan 54 \\
\hline M8 & Yitpi \\
\hline
\end{tabular}


Table S2: Homology search (BLASTp analysis) of ATI peptides from wheat cultivars monitored by sMRM experiment. Peptides marked as $(*)$ asterisk are plotted in Figure 3 and 5.

\begin{tabular}{|c|c|c|c|c|c|}
\hline Accession & Peptide Sequence & Name & BLASTp results & Unique & $100 \%$ of Identity match in NCBI \\
\hline P16347 & ADANYYVLPANR & $\begin{array}{l}\text { Endogenous alpha- } \\
\text { amylase/subtilisin } \\
\text { inhibitor }\end{array}$ & P16347, A0A1D5UPZ8, R7W4S0 & No & $\begin{array}{l}\text { Triticum aestivum, Aegilops } \\
\text { tauschii }\end{array}$ \\
\hline P16347 & AHGGGLTMAPGHGR & $\begin{array}{l}\text { Alpha- } \\
\text { amylase/subtilisin } \\
\text { inhibitor }\end{array}$ & $\begin{array}{l}\text { P07596, P16347, A0A1D5TYI6, A0A1D5TD52, } \\
\text { F2E8J4, J9V7J5, A0A1D5UPZ8, R7W4S0 }\end{array}$ & No & $\begin{array}{l}\text { Hordeum vulgare, Triticum } \\
\text { aestivum, Hordeum vulgare f. } \\
\text { agriocrit, Aegilops tauschii }\end{array}$ \\
\hline P16347 & CPLFVSQEADGQR & $\begin{array}{l}\text { Endogenous alpha- } \\
\text { amylase/subtilisin } \\
\text { inhibitor }\end{array}$ & $\begin{array}{l}\text { P16347, A0A1D5TYI6, A0A1D5TD52, } \\
\text { A0A1D5UPZ8, R7W4S0 }\end{array}$ & No & $\begin{array}{l}\text { Triticum aestivum, Aegilops } \\
\text { tauschii }\end{array}$ \\
\hline P16347 & HVITGPVR & $\begin{array}{l}\text { Endogenous alpha- } \\
\text { amylase/subtilisin } \\
\text { inhibitor }\end{array}$ & $\begin{array}{l}\text { P16347, A0A1D5TYI6, A0A1D5TD52, } \\
\text { A0A1D5UPZ8 }\end{array}$ & No & $\begin{array}{l}\text { Triticum aestivum, Aegilops } \\
\text { tauschii }\end{array}$ \\
\hline P16347 & LMACGDSCQDLGVFR & $\begin{array}{l}\text { Endogenous alpha- } \\
\text { amylase/subtilisin } \\
\text { inhibitor }\end{array}$ & P16347, A0A1D5TYI6, A0A1D5TD52, M7ZF09 & No & Triticum aestivum, Triticum urartu \\
\hline P16347 & YSGAEVHEYK & $\begin{array}{l}\text { Endogenous alpha- } \\
\text { amylase/subtilisin } \\
\text { inhibitor }\end{array}$ & $\begin{array}{l}\text { P07596, P16347, A0A1D5TYI6, A0A1D5TD52, } \\
\text { F2E8J4, J9V7J5, A0A287IWR2 }\end{array}$ & No & $\begin{array}{l}\text { Hordeum vulgare, Triticum } \\
\text { aestivum, Hordeum vulgare f. } \\
\text { agriocrit, Aegilops tauschii }\end{array}$ \\
\hline Q5UHH7 & CGALYSMLDSMYK & $\begin{array}{l}0.19 \text { dimeric alpha- } \\
\text { amylase inhibitor }\end{array}$ & $\begin{array}{l}\text { P01084, P01085, Q5UHH7, A0A1D5VPJ2, } \\
\text { M8BUP2, W5D003, A0A1D5WWL8, W5DK32, } \\
\text { M8C3B8, A0A077RSX3, A0A077RQ83, } \\
\text { A0A1D5WX53, C3VWV8, C3VWV0, C3VWU9, } \\
\text { C3VWV3 }\end{array}$ & No & $\begin{array}{l}\text { Triticum aestivum, Aegilops } \\
\text { tauschii, Secale cereale, } \\
\text { Eremopyrum bonaepartis }\end{array}$ \\
\hline Q5UHH7 & DCCQQLAHISEWCR & $\begin{array}{l}0.19 \text { dimeric alpha- } \\
\text { amylase inhibitor }\end{array}$ & $\begin{array}{l}\text { P01084, P01085, Q5UHH7, A0A1D5VPJ2, } \\
\text { M8BUP2, W5D003, A0A1D5WWL8, W5DK32, } \\
\text { M8C3B8, A0A077RSX3, A0A077RQ83, } \\
\text { A0A1D5WX53, C3VWV8, C3VWV0, C3VWU9, } \\
\text { C3VWV3, C3VX00, C3VWZ9, C3VWT8, } \\
\text { C3VWZ1, Q4U1A2, C8CAI4, Q4U196, I6PZ03, } \\
\text { C3VWW9, C3VWW8, C3VWX8, C3VWU5, } \\
\text { C3VWS2, C3VWT7, C3VWE2, C3VWL6, } \\
\text { C3VWU3, C3VWX6, C3VX03, C3VX04, } \\
\text { C3VWW4, A0A1D5WWL9, L7P101, L7P0L4, }\end{array}$ & No & $\begin{array}{l}\text { Triticum aestivum, Aegilops } \\
\text { tauschii, Secale cereale, } \\
\text { Eremopyrum bonaepartis }\end{array}$ \\
\hline
\end{tabular}




\begin{tabular}{|c|c|c|c|c|c|}
\hline & & & $\begin{array}{l}\text { L7P100, M1NYK0, L7P0Z6, L7P0N7, L7P0X6, } \\
\text { M1PM40, L7P0X2, Q19A44, Q5UHI0, A4GFU5, } \\
\text { A4GFU7, A4GFU1, Q5UHH1, A4GFU4, Q5UHH3, } \\
\text { Q4W0V7, C3VWZ8, Q5UHH4 }\end{array}$ & & \\
\hline Q5UHH7 & "EHGAQEGQAGTGAFPR & $\begin{array}{l}0.19 \text { dimeric alpha- } \\
\text { amylase inhibitor }\end{array}$ & $\begin{array}{l}\text { P01085, Q5UHH7, A0A341RUL7, A0A1D5VPJ2, } \\
\text { M8BUP2, W5D003, A0A1D5WWL8, W5DK32, } \\
\text { M8C3B8, A0A077RSX3, A0A077RQ83, } \\
\text { A0A1D5WX53, C3VWV8, C3VWV0, C3VWU9, } \\
\text { C3VWV4, C3VWZ1, C3VX00, C3VWT8, } \\
\text { C3VWN1, C3VWJ7, C3VWH6, C3VWK6, } \\
\text { C3VWR4, C3VWL9, C3VWP8, C3VW62, } \\
\text { C3VWH3, C3VWH8, C3VWR5, C3VW81, } \\
\text { C3VWJ0, C3VWD4, C3VWG4, C3VW64, } \\
\text { C3VWL8, C3VWE9, C3VWR3, C3VWE5, } \\
\text { C3VWI7, C3VWF4, C3VWG0, C3VWJ1, C3VWP7, } \\
\text { Q4U1A2, Q4U1A0, C8CAI4, Q4U196, I6PZ03, } \\
\text { C3VWX1, C3VWW9, C3VWW8, C3VWX8, } \\
\text { C3VWU6, C3VWU5, C3VWW6, C3VWW3, } \\
\text { C3VWS4, C3VWR8, C3VWS0, C3VWR8, } \\
\text { C3VWS2, C3VWT7, C3VWR7, C3VW87, } \\
\text { C3VWE2, C3VWL6, C3VW61, C3VWU3, } \\
\text { C3VWX6, C3VX03, C3VX04, C3VWW4, C3VX01, } \\
\text { C3VW79, C3VW86, S4UM47, A0A1D5VKI2, } \\
\text { A0A1D5WWL9, I6Q083, L7P101, L7P0X4, } \\
\text { L7P0Z7, L7P0L4, L7P100, M1NYK0, L7P0Z6, } \\
\text { L7P0N7, L7P0X6, M1PM40, L7P0X2, Q19A44, } \\
\text { Q5UHI0, A4GFU5, A4GFR3, A4GFU1, Q5UHH1, } \\
\text { A4GFU4, Q5UHH7, Q5UHH3, Q4W0V7, C3VWZ8 }\end{array}$ & No & $\begin{array}{l}\text { Triticum aestivum, Aegilops } \\
\text { tauschii, Secale cereale, } \\
\text { Eremopyrum bonaepartis, } \\
\text { Hordeum vulgare }\end{array}$ \\
\hline Q5UHH7 & "LPIVVDASGDGAYVCK & $\begin{array}{l}0.19 \text { dimeric alpha- } \\
\text { amylase inhibitor }\end{array}$ & $\begin{array}{l}\text { A0A1D5WWL8, W5DK32, M8C3B8, } \\
\text { A0A077RSX3, A0A077RQ83, A0A1D5WX53, } \\
\text { C3VWW0, C3VWW1, C3VWV8, C3VWV4, } \\
\text { C3VWZ8, C3VX00, C3VWZ9, C3VWT8, } \\
\text { C3VWC7, C3VWL1, C3VWR4, C3VWR1, } \\
\text { C3VWJ3, C3VWJ8, C3VWL3, C3VWH8, C3VW70, } \\
\text { C3VWR5, C3VWE6, C3VWJ0, C3VW96, } \\
\text { C3VWC3, C3VWD7, C3VWQ2, C3VWH7, } \\
\text { C3VWD9, C3VWC5, C3VWQ9, C3VW99, } \\
\text { C3VWQ7, C3VWZ2, C3VWR3, C3VWZ1, } \\
\text { C3VWZ5, C3VWF1, C3VWP3, C3VWA4, }\end{array}$ & No & $\begin{array}{l}\text { Triticum aestivum, Aegilops } \\
\text { tauschii, Secale cereale, } \\
\text { Eremopyrum bonaepartis, } \\
\text { Hordeum vulgare }\end{array}$ \\
\hline
\end{tabular}




\begin{tabular}{|c|c|c|c|c|c|}
\hline & & & $\begin{array}{l}\text { C3VWJ9, Q4U198, Q4U1A5, Q4U1A4, Q4U1A2, } \\
\text { Q4U1A0, Q4U196, Q4U197, I6PZ03, } \\
\text { A0A1D5VPJ3, C3VX09, C3VX10, C3VWY8, } \\
\text { C3VWY7, C3VWX1, C3VWW9, C3VWW8, } \\
\text { C3VWX8, C3VWU6, C3VWU5, C3VWW2, } \\
\text { C3VWS2, C3VWT2, C3VWT7, C3VWY2, } \\
\text { C3VWY5, C3VWZ0, C3VWQ4, C3VW77, } \\
\text { C3VWV2, C3VWE2, C3VWL6, C3VW78, } \\
\text { C3VW69, C3VWU3, C3VWX6, C3VX03, C3VX04, } \\
\text { C3VWV4, A0A1D5WWL9, L7P101, L7P0X4, } \\
\text { L7P0Z7, L7P0L4, L7P100, L7P0Z6, L7P0N7, } \\
\text { M8BUP2, L7P0X2, L7P0L8, Q19A45, Q19A44, } \\
\text { A4GFW9, A4GFU9, A4GFW3, A4GFV1, A4GFV0, } \\
\text { A4GFX0, A4GFX4, A4GFW2, A4GFX3, A4GFV7, } \\
\text { A4GFR7, A4GFR9, A4GFR6, A4GFS, A4GFR8, } \\
\text { A4GFX6, A4GFZ4, A4GFP6, A4GFR0, A4GFR1, } \\
\text { A4GFR2, A4GFQ9, A4GFP7, Q5UH1, Q5MD68, } \\
\text { A4GG07, A4GG12, A4GG03, A4GFT1, A4GFU0, } \\
\text { A4GFT5, A4GFS4, A4GFT2, A4GFU7, A4GFT4, } \\
\text { A4GFS3, A4GFU1, A4GFS7, A4GFT7, Q5UHH4, } \\
\text { Q5UHH1, L7P0N5, A5HE08, A5HE29, A5HDZ8, } \\
\text { A5HE07, A5HE18, A5HE22, A5HDZ6, A4GFR4, } \\
\text { A4GFR3, A4GFV6, A4GFU8, A4GFY6, A4GFS5, } \\
\text { A4GFP2, Q5UHH7, A4GG16, A4GG01, A4GG09, } \\
\text { A4GG14, Q5UHH3, A4GG04, A4GFZ9, Q5I4D7, } \\
\text { A5HE16, A5HE12, A5HDZ5, P01084, P01085, } \\
\text { M1PYS5, Q4W0V7, I6PWK7 }\end{array}$ & & \\
\hline Q5UHH7 & LQCNGSQVPEAVLR & $\begin{array}{l}0.19 \text { dimeric alpha- } \\
\text { amylase inhibitor }\end{array}$ & $\begin{array}{l}\text { P10846, A0A1D5VPJ2, M8BUP2, A0A1D5WWL8, } \\
\text { W5D003, W5DK32, M8C3B8, A0A077RSX3, } \\
\text { A0A077RQ83, C3VX13, C3VWW0, C3VWW1, } \\
\text { C3VWV8, T1WHS5, T1WHQ5, C3VWZ6, } \\
\text { T1WIK9, T1WH90, T1WI05, C3VWZ8, C3VX00, } \\
\text { C3VWZ9, C3VWT8, C3VWC7, C3VWL1, } \\
\text { C3VWJ7, C3VWK6, C3VWR4, C3VWR1, } \\
\text { C3VWJ4, C3VWM4, C3VWQ4, C3VWJ3, } \\
\text { C3VWJ8, C3VW67, C3VWL3, C3VWH8, } \\
\text { C3VW70, C3VWR5, C3VWE6, C3VWJ0, } \\
\text { C3VWB8, C3VWN4, C3VWY9, C3VW96, } \\
\text { C3VWD7, C3VWP0, C3VWQ2, C3VWH7, }\end{array}$ & No & $\begin{array}{l}\text { Triticum aestivum, Aegilops } \\
\text { tauschii, Secale cereale, } \\
\text { Eremopyrum bonaepartis, } \\
\text { Hordeum vulgare, Thinopyrum } \\
\text { bessarabicum }\end{array}$ \\
\hline
\end{tabular}




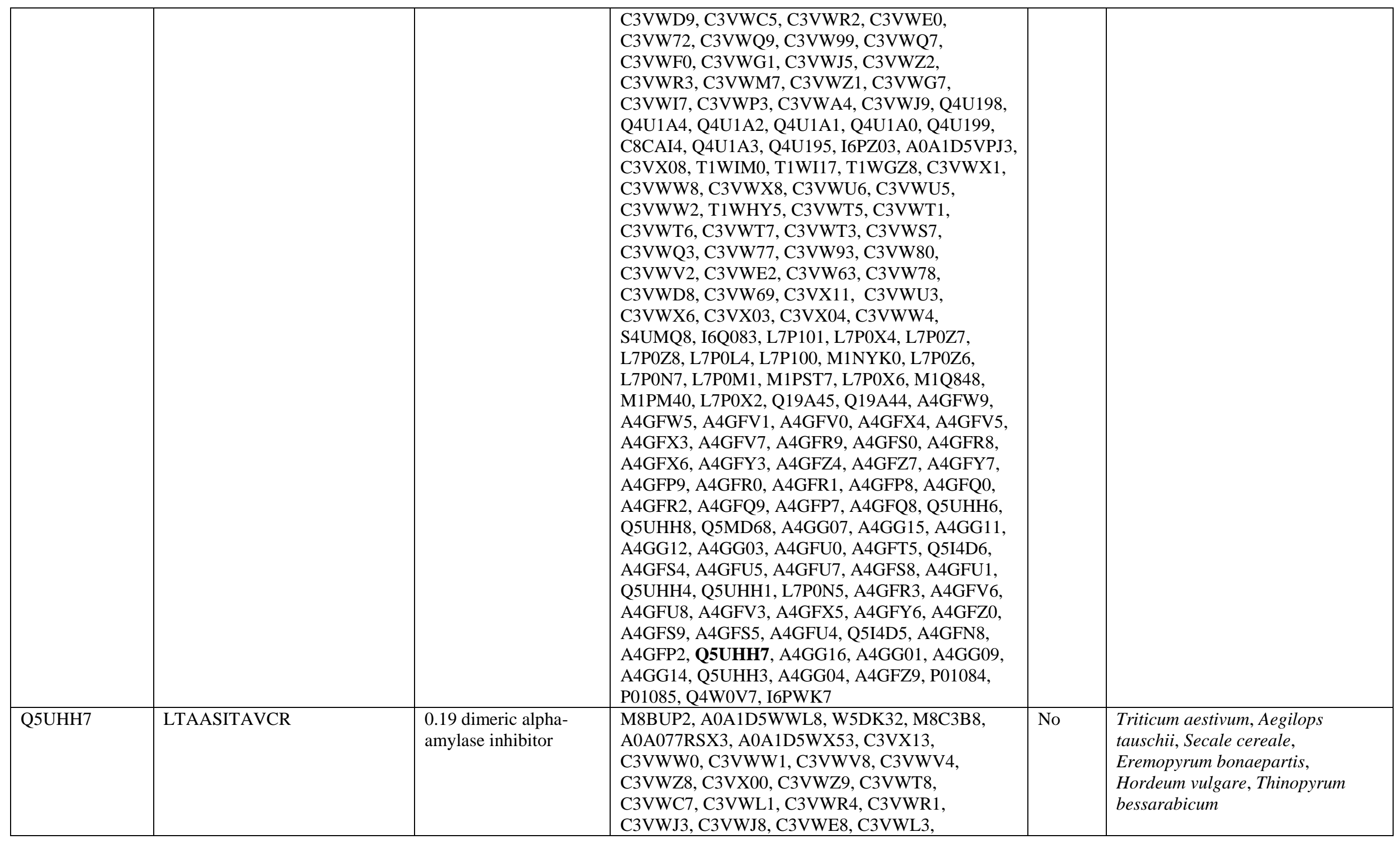




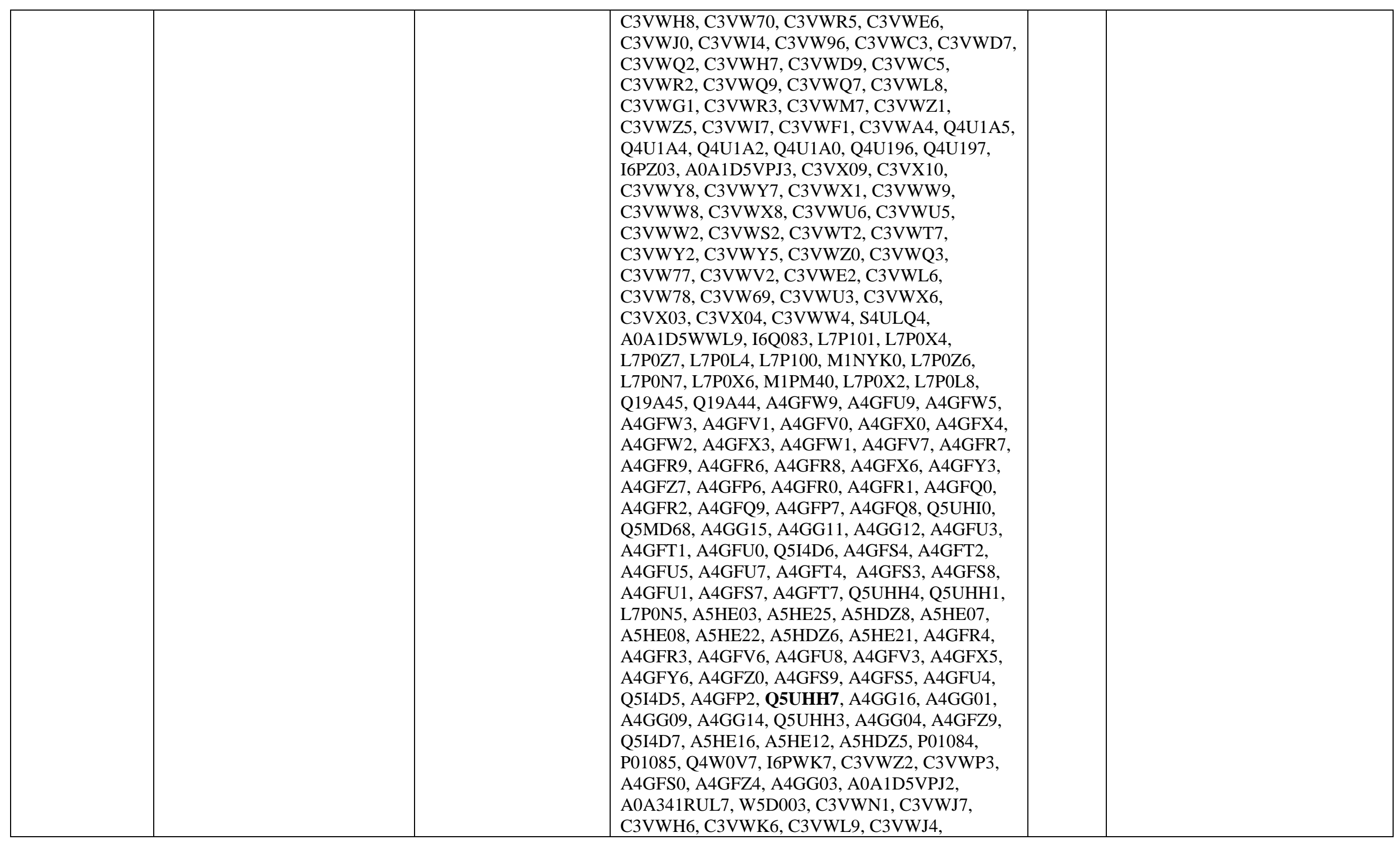




\begin{tabular}{|c|c|c|c|c|c|}
\hline & & & $\begin{array}{l}\text { C3VWM4, C3VWQ4, C3VWP8, C3VW62, } \\
\text { C3VW67, C3VWH3, C3VWA5, C3VW81, } \\
\text { C3VWP9, C3VWB5, C3VWD4, C3VWN4, } \\
\text { C3VWY9, C3VWP0, C3VWG4, C3VW64, } \\
\text { C3VWE0, C3VW99, C3VWF7, C3VWF0, C3VWJ5, } \\
\text { C3VWE9, C3VWE5, C3VWG7, C3VWG2, } \\
\text { C3VWF4, C3VWG0, C3VWA8, C3VWJ1, } \\
\text { C3VWZ4, C3VWP7, Q4U198, Q4U1A1, Q4U199, } \\
\text { C8CAI4, Q4U1A3, Q4U195, C3VX08, C3VWW6, } \\
\text { C3VWW3, C3VWS4, C3VWT5, C3VWT1, } \\
\text { C3VWT3, C3VWS0, C3VWR8, C3VWT6, } \\
\text { C3VWR7 }\end{array}$ & & \\
\hline \multirow[t]{2}{*}{ C4P622 } & ${ }^{*}$ CGDLSSMLR & $\begin{array}{l}\text { Alpha-amylase } \\
\text { inhibitor } 0.28\end{array}$ & $\begin{array}{l}\text { P01083, C4P661, C4P5M9, C4P5V7, C4P5G2, } \\
\text { C4P5X2, C4P5Y7, C4P5I, C4P5D0, C4P5W2, } \\
\text { C4P5N6, C4P550, C4P5N1, C4P5F8, C4P5Z0, } \\
\text { C4P5C2, C4P5L9, C4P5P7, C4P5I4, C4P5K4, } \\
\text { C4P652, C4P5E1, C4P5J9, C4P681, C4P5K2, } \\
\text { C4P5I8, C4P5V6, C4P5G9, C4P5C4, C4P5W5, } \\
\text { C4P627, C4P615, C4P5W7, C4P5U8, C4P655, } \\
\text { C4P5C9, C4P5E7, C4P5Y9, C4P622, C4P5R2, } \\
\text { C4P5B9, C4P5X6, C4P684, C4P639, C4P5H8, } \\
\text { C4P5J2, C4P5A6, C4P5Z6, C4P678, C4P5R3, } \\
\text { C4P5R6, C4P5D2, C4P5H6, C4P673, C4P5L2, } \\
\text { C4P586, C4P5A2, C4P633, C4P5B7, C4P647, } \\
\text { C4P5E5, C4P598, C4P5G0, C4P5Z1, C4P594, } \\
\text { C4P5B6, C4P5S3, C4P617, C4P5N2, C4P5A1, } \\
\text { C4P5G4, C4P5W6, C4P5E4, C4P623, C4P5D4, } \\
\text { C4P665, C4P5C1, C4P5I2, C4P5J4, C4P629, } \\
\text { C4P591, C4P5F3, C4P593, C4P649, C4P5F6, } \\
\text { C4P5U2, C4P599, C4P584, C4P583, X2KYP9, } \\
\text { R7W9C2, C4P5R8, C3VWV7, A4ZIY9, A4ZIX1, } \\
\text { A4ZJ03, A4ZIW5, A4ZIV1, A4ZIV2, A4ZIV5, } \\
\text { A4ZIW9, A4ZIZ0, A4ZIZ6, A4ZIW6, A4ZIV8, } \\
\text { A4ZIU3, A4ZIT6, A4ZIU6, A4ZIU7, } \\
\text { A0A1D6BDS7, A0A1D6BDS6 }\end{array}$ & No & $\begin{array}{l}\text { Triticum aestivum, Triticum } \\
\text { dicoccoides, Aegilops tauschii, } \\
\text { Triticum monococcum, Aegilops } \\
\text { bicornis, Aegilops longissima }\end{array}$ \\
\hline & "DCCQQLADINNEWCR & $\begin{array}{l}\text { Alpha-amylase } \\
\text { inhibitor } 0.28\end{array}$ & $\begin{array}{l}\text { P01083, C4P661, C4P5M9, C4P5V7, C4P5G2, } \\
\text { C4P5Y7, C4P519, C4P5D0, C4P5S0, C4P5N1, } \\
\text { C4P5F8, C4P5Z0, C4P5C2, C4P5P7, C4P5K4, } \\
\text { C4P652, C4P5E1, C4P681, C4P5K2, C4P5I8, }\end{array}$ & No & $\begin{array}{l}\text { Triticum aestivum, Triticum } \\
\text { dicoccoides, Aegilops tauschii, } \\
\text { Triticum monococcum, Aegilops } \\
\text { bicornis, Aegilops longissima }\end{array}$ \\
\hline
\end{tabular}




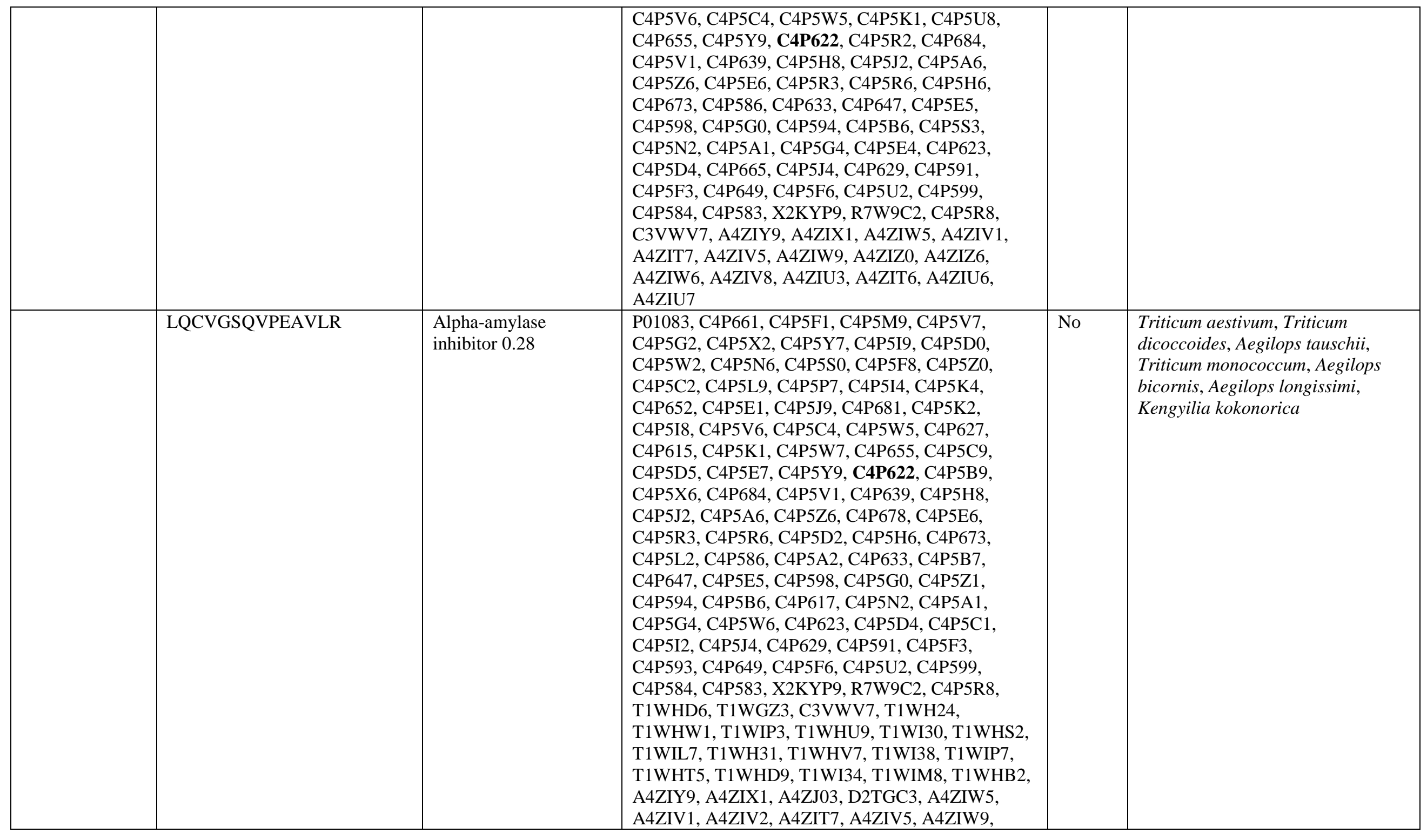




\begin{tabular}{|c|c|c|c|c|}
\hline & & $\begin{array}{l}\text { A4ZIZ0, A4ZIZ6, A4ZIW6, A4ZIV8, A4ZIU3, } \\
\text { A4ZIT6, A4ZIU6, A4ZIU7 }\end{array}$ & & \\
\hline LTAASVPEVCK & $\begin{array}{l}\text { Alpha-amylase } \\
\text { inhibitor } 0.28\end{array}$ & $\begin{array}{l}\text { P01083, C4P661, C4P5F1, C4P5M9, C4P5V7, } \\
\text { C4P5G2, C4P5X2, C4P5Y7, C4P5D0, C4P5W2, } \\
\text { C4P5N6, C4P5S0, C4P5N1, C4P5Z0, C4P5C2, } \\
\text { C4P5L9, C4P5P7, C4P5I4, C4P5K4, C4P652, } \\
\text { C4P5E1, C4P5J9, C4P681, C4P5I8, C4P5G9, } \\
\text { C4P5C4, C4P5W5, C4P627, C4P615, C4P5W7, } \\
\text { C4P5U8, C4P5C9, C4P5D5, C4P5Y9, C4P622, } \\
\text { C4P5R2, C4P5B9, C4P5X6, C4P684, C4P5V1, } \\
\text { C4P639, C4P5H8, C4P5J2, C4P5A6, C4P5E6, } \\
\text { C4P5R3, C4P5R6, C4P5D2, C4P5H6, C4P5L2, } \\
\text { C4P586, C4P5A2, C4P5B7, C4P647, C4P5E5, } \\
\text { C4P598, C4P5Z1, C4P5B6, C4P5S3, C4P617, } \\
\text { C4P5N2, C4P5A1, C4P5G4, C4P5W6, C4P5E4, } \\
\text { C4P623, C4P5D4, C4P665, C4P5C1, C4P5I2, } \\
\text { C4P5J4, C4P591, C4P5F3, C4P593, C4P649, } \\
\text { C4P5F6, C4P5U2, C4P599, C4P584, C4P583, } \\
\text { X2KYP9, R7W9C2, C4P5R8, T1WHR6, C3VWV7, } \\
\text { T1WHW1, T1WH20, T1WIN9, T1WHC3, } \\
\text { T1WHE4, T1WIP7, T1WHD2, T1WH04, T1WI34, } \\
\text { T1WHZ6, T1WH99, T1WIL2, T1WH95, T1WIM5, } \\
\text { T1WI21, A4ZIX1, A4ZJ03, A4ZIW5, A4ZIV2, } \\
\text { A4ZIT7, A4ZIV5, A4ZIW9, A4ZIZ0, A4ZIZ6, } \\
\text { A4ZIW6, A4ZIV8, A4ZIU3, A4ZIT6, A4ZIU6, } \\
\text { A4ZIU7, A0A1D6BDS7, A0A1D6BDS6, C4P5V6 }\end{array}$ & No & $\begin{array}{l}\text { Triticum aestivum, Triticum } \\
\text { dicoccoides, Aegilops tauschii, } \\
\text { Triticum monococcum, Aegilops } \\
\text { bicornis, Aegilops longissimi, } \\
\text { Kengyilia kokonorica }\end{array}$ \\
\hline SVYQELGVR & $\begin{array}{l}\text { Alpha-amylase } \\
\text { inhibitor } 0.28\end{array}$ & $\begin{array}{l}\text { P01083, C4P661, C4P5F1, C4P5M9, C4P5G2, } \\
\text { C4P5X2, C4P5Y7, C4P5I9, C4P5D0, C4P5W2, } \\
\text { C4P5N6, C4P5S0, C4P5N1, C4P5F8, C4P5Z0, } \\
\text { C4P5C2, C4P5L9, C4P5P7, C4P5I4, C4P5K4, } \\
\text { C4P5J9, C4P681, C4P5K2, C4P5I8, C4P5V6, } \\
\text { C4P5G9, C4P5C4, C4P5W5, C4P627, C4P615, } \\
\text { C4P5K1, C4P5W7, C4P5U8, C4P655, C4P5C9, } \\
\text { C4P5D5, C4P5E7, C4P5Y9, C4P622, C4P5R2, } \\
\text { C4P5B9, C4P5X6, C4P684, C4P5V1, C4P639, } \\
\text { C4P5J2, C4P5A6, C4P5Z6, C4P678, C4P5E6, } \\
\text { C4P5R3, C4P5R6, C4P5D2, C4P5H6, C4P673, } \\
\text { C4P5L2, C4P586, C4P5A2, C4P633, C4P5B7, } \\
\text { C4P647, C4P5E5, C4P598, C4P5G0, C4P5Z1, }\end{array}$ & No & $\begin{array}{l}\text { Triticum aestivum, Triticum } \\
\text { dicoccoides, Aegilops tauschii, } \\
\text { Triticum monococcum, Aegilops } \\
\text { bicornis, Aegilops longissimi, } \\
\text { Kengyilia kokonorica }\end{array}$ \\
\hline
\end{tabular}




\begin{tabular}{|c|c|c|c|c|}
\hline & & $\begin{array}{l}\text { C4P594, C4P5B6, C4P5S3, C4P617, C4P5N2, } \\
\text { C4P5A1, C4P5G4, C4P5W6, C4P5E4, C4P623, } \\
\text { C4P5D4, C4P5C1, C4P5I2, C4P5J4, C4P629, } \\
\text { C4P591, C4P5F3, C4P593, C4P649, C4P5F6, } \\
\text { C4P5U2, C4P599, C4P584, C4P583, X2KYP9, } \\
\text { R7W9C2, C3VWV7, A4ZIY9, A4ZIX1, A4ZJ03, } \\
\text { A4ZIV1, A4ZIV2, A4ZIT7, A4ZIV5, A4ZIW9, } \\
\text { A4ZIZ0, A4ZIZ6, A4ZIW6, A4ZIV8, A4ZIU3, } \\
\text { A4ZIT6, A4ZIU6, A4ZIU7, A0A1D6BDS7, } \\
\text { A0A1D6BDS6 }\end{array}$ & & \\
\hline VPIPNPSGDR & $\begin{array}{l}\text { Alpha-amylase } \\
\text { inhibitor } 0.28\end{array}$ & $\begin{array}{l}\text { P01083, C4P5G2, C4P5X2, C4P5N6, C4P5N1, } \\
\text { C4P5Z0, C4P5I4, C4P5K4, C4P652, C4P5J9, } \\
\text { C4P681, C4P5K2, C4P5G9, C4P627, C4P615, } \\
\text { C4P5W7, C4P5D5, C4P5Y9, C4P622, C4P5B9, } \\
\text { C4P684, C4P5D2, C4P5L2, C4P5Z1, C4P617, } \\
\text { C4P5W6, C4P5D4, C4P5C1, C4P5I2, C4P591, } \\
\text { C4P5F3, C4P593, C4P649, X2KYP9, R7W9C2, } \\
\text { A4ZIY9, A4ZIX1, D2TGC3, A4ZIW5, A4ZIV1, } \\
\text { A4ZIV2, A4ZIT7, A4ZIV5, A4ZIW9, A4ZIZ6, } \\
\text { A4ZIW6, A4ZIV8, A4ZIU3, A4ZIT6, A4ZIU6, } \\
\text { A4ZIU7, A0A1D6BDS7, A0A1D6BDS6, C4P678, } \\
\text { C4P661, C4P5F1, C4P5M9, C4P5V7, C4P5Y7, } \\
\text { C4P5I9, C4P5D0, C4P5W2, C4P5S0, C4P5F8, } \\
\text { C4P5C2, C4P5L9, C4P5P7, C4P5E1, C4P5I8, } \\
\text { C4P5V6, C4P5C4, C4P5W5, C4P5K1, C4P5U8, } \\
\text { C4P655, C4P5C9, C4P5E7, C4P5R2, C4P5X6, } \\
\text { C4P5V1, C4P639, C4P5J2, C4P5A6, C4P5Z6, } \\
\text { C4P5E6, C4P5R3, C4P5R6, C4P5H6, C4P673, } \\
\text { C4P586, C4P5A2, C4P633, C4P5B7, C4P647, } \\
\text { C4P5E5, C4P598, C4P5G0, C4P594, C4P5B6, } \\
\text { C4P5S3, C4P5N2, C4P5A1, C4P5E4, C4P623, } \\
\text { C4P665, C4P5J4, C4P629, C4P5F6, C4P5U2, } \\
\text { C4P599, C4P584, C4P583, C4P5R8, A4ZJ03, } \\
\text { A4ZIZ0 }\end{array}$ & No & $\begin{array}{l}\text { Triticum aestivum, Triticum } \\
\text { dicoccoides, Aegilops tauschii, } \\
\text { Triticum monococcum, Aegilops } \\
\text { bicornis, Aegilops longissimi, } \\
\text { Kengyilia kokonorica }\end{array}$ \\
\hline VVSALTGCR & $\begin{array}{l}\text { Alpha-amylase } \\
\text { inhibitor } 0.28\end{array}$ & $\begin{array}{l}\text { P01083, C4P661, C4P5N1, C4P622, C4P5L9, } \\
\text { C4P5F8, C4P5C2, C4P5D0, C4P5P7, C4P5W2, } \\
\text { C4P5I4, C4P5Z0, C4P5Y7, C4P5I9, C4P5X2, } \\
\text { C4P5G2, C4P5M9 }\end{array}$ & No & $\begin{array}{l}\text { Triticum aestivum, Triticum } \\
\text { dicoccoides, Aegilops tauschii, } \\
\text { Triticum monococcum, Aegilops } \\
\text { bicornis, Aegilops longissimi, } \\
\text { Kengyilia kokonorica }\end{array}$ \\
\hline
\end{tabular}




\begin{tabular}{|c|c|c|c|c|c|}
\hline \multirow[t]{7}{*}{ Q6S5B1 } & DYVLQQTCGTFTPGSK & $\begin{array}{l}\text { Alpha-amylase/trypsin } \\
\text { inhibitor CM3 }\end{array}$ & $\begin{array}{l}\text { P17314, M8BV45, Q53YX8, Q6S5B1, } \\
\text { A0A1D5XLP1, A0A1D5Y0V2, A0A1S6KXP9 }\end{array}$ & Yes & $\begin{array}{l}\text { Triticum aestivum, Aegilops } \\
\text { tauschii, Triticum turgidum subsp. } \\
\text { durum }\end{array}$ \\
\hline & EMQWDFVR & $\begin{array}{l}\text { Alpha-amylase/trypsin } \\
\text { inhibitor CM3 }\end{array}$ & P17314, Q53YX8, Q6S5B1 & Yes & $\begin{array}{l}\text { Triticum aestivum, Triticum } \\
\text { turgidum subsp. durum }\end{array}$ \\
\hline & LLVAPGQCNLATIHNVR & $\begin{array}{l}\text { Alpha-amylase/trypsin } \\
\text { inhibitor CM3 }\end{array}$ & $\begin{array}{l}\text { P11643, P17314, M8BV45, O23982, O24000, } \\
\text { M0Y227, Q53YX8, Q6S5B1, A0A1S6KXP9 }\end{array}$ & No & $\begin{array}{l}\text { Hordeum vulgare, Triticum } \\
\text { aestivum, Aegilops tauschii, } \\
\text { Triticum turgidum subsp. durum }\end{array}$ \\
\hline & LYCCQELAEISQQCR & $\begin{array}{l}\text { Alpha-amylase/trypsin } \\
\text { inhibitor CM3 }\end{array}$ & $\begin{array}{l}\text { P17314, Q53YX8, Q6S5B1, A0A1D5XLP1, } \\
\text { A0A1S6KXP9 }\end{array}$ & Yes & $\begin{array}{l}\text { Triticum aestivum, Triticum } \\
\text { turgidum subsp. durum }\end{array}$ \\
\hline & SGNVGESGLIDLPGCPR & $\begin{array}{l}\text { Alpha-amylase/trypsin } \\
\text { inhibitor CM3 }\end{array}$ & $\begin{array}{l}\text { P17314, M8BV45, Q53YX8, Q6S5B1, } \\
\text { A0A1S6KXP9 }\end{array}$ & Yes & $\begin{array}{l}\text { Triticum aestivum, Aegilops } \\
\text { tauschii, Triticum turgidum subsp. } \\
\text { durum }\end{array}$ \\
\hline & TNLLPHCR & $\begin{array}{l}\text { Alpha-amylase/trypsin } \\
\text { inhibitor CM3 }\end{array}$ & $\begin{array}{l}\text { P17314, Q53YX8, Q6S5B1, A0A1D5XLP1, } \\
\text { A0A1S6KXP9 }\end{array}$ & Yes & $\begin{array}{l}\text { Triticum aestivum, Triticum } \\
\text { turgidum subsp. durum }\end{array}$ \\
\hline & YFIALPVPSQPVDPR & $\begin{array}{l}\text { Alpha-amylase/trypsin } \\
\text { inhibitor CM3 }\end{array}$ & $\begin{array}{l}\text { P17314, Q53YX8, Q6S5B1, A0A1D5XLP1, } \\
\text { A0A1S6KXP9 }\end{array}$ & Yes & $\begin{array}{l}\text { Triticum aestivum, Triticum } \\
\text { turgidum subsp. durum }\end{array}$ \\
\hline \multirow[t]{5}{*}{ R7W9W1 } & ${ }^{*}$ DLPGCPR & $\begin{array}{l}\text { Alpha-amylase/trypsin } \\
\text { inhibitor CM2 }\end{array}$ & Short sequence. Unable to match any Uniprot ID & N/A & N/A \\
\hline & ${ }^{*}$ ELYDASQHCR & $\begin{array}{l}\text { Alpha-amylase/trypsin } \\
\text { inhibitor CM2 }\end{array}$ & $\begin{array}{l}\text { P16851, P16850, A0A1D6CFU7, A0A1D6D8Z1, } \\
\text { R7W9W1, C7C4X0, D2TGC2, }\end{array}$ & Yes & $\begin{array}{l}\text { Triticum aestivum, Aegilops } \\
\text { tauschii }\end{array}$ \\
\hline & $\begin{array}{l}\text { EYVAQQTCGISISGSAVSTEP } \\
\text { GNTPR }\end{array}$ & $\begin{array}{l}\text { Alpha-amylase/trypsin } \\
\text { inhibitor CM1 }\end{array}$ & P16850, C7C4X0, A0A1D6D8Z1, R7W9W1 & Yes & $\begin{array}{l}\text { Triticum aestivum, Aegilops } \\
\text { tauschii }\end{array}$ \\
\hline & SDPNSSVLK & $\begin{array}{l}\text { Alpha-amylase/trypsin } \\
\text { inhibitor CM1 }\end{array}$ & P16850, C7C4X0, A0A1D6D8Z1, R7W9W1 & Yes & $\begin{array}{l}\text { Triticum aestivum, Aegilops } \\
\text { tauschii }\end{array}$ \\
\hline & $\begin{array}{l}\text { VLVTPGHCNVMTVHNAPY } \\
\text { CLGLDI }\end{array}$ & $\begin{array}{l}\text { Alpha-amylase/trypsin } \\
\text { inhibitor CM1 }\end{array}$ & C7C4X0, A0A1D6D8Z1, R7W9W1 & Yes & $\begin{array}{l}\text { Triticum aestivum, Aegilops } \\
\text { tauschii }\end{array}$ \\
\hline \multirow[t]{3}{*}{ Q5UHH6 } & ECCQQLADISEWCR & $\begin{array}{l}0.19 \text { dimeric alpha- } \\
\text { amylase inhibitor }\end{array}$ & $\begin{array}{l}\text { W5D003, Q4U1A1, Q4U199, Q4U1A3, Q4U195, } \\
\text { C3VX08, C3VX11, Q5UHH6, Q5UHH8 }\end{array}$ & No & $\begin{array}{l}\text { Triticum aestivum, Thinopyrum } \\
\text { bessarabicum }\end{array}$ \\
\hline & EHGVQEGQAGTGAFPSCR & $\begin{array}{l}0.19 \text { dimeric alpha- } \\
\text { amylase inhibitor }\end{array}$ & $\begin{array}{l}\text { W5D003, C3VWN4, C3VWP0, C3VWP9, } \\
\text { C3VWM4, C3VWF0, C3VWJ5, Q5UHH6, } \\
\text { Q5UHH8, L7P0M1 }\end{array}$ & No & $\begin{array}{l}\text { Triticum aestivum, Triticum } \\
\text { dicoccoides, Henrardia persica, } \\
\text { Triticum timopheevii, Thinopyrum } \\
\text { bessarabicum }\end{array}$ \\
\hline & LPIVIDASGDGAYVCK & $\begin{array}{l}0.19 \text { dimeric alpha- } \\
\text { amylase inhibitor }\end{array}$ & $\begin{array}{l}\text { W5D003, T1WHZ1, T1WIJ6, T1WHQ5, T1WHU0, } \\
\text { C3VWV0, C3VWV3, C3VWZ7, Q5UHH6, } \\
\text { C3VWZ6 }\end{array}$ & No & $\begin{array}{l}\text { Triticum aestivum, Kengyilia } \\
\text { batalinii, Agropyron cristatum, } \\
\text { Agropyron mongolicum, } \\
\text { Eremopyrum bonaepartis, Triticum } \\
\text { dicoccoides }\end{array}$ \\
\hline
\end{tabular}




\begin{tabular}{|c|c|c|c|c|c|}
\hline & LTAASITAVCK & $\begin{array}{l}0.19 \text { dimeric alpha- } \\
\text { amylase inhibitor }\end{array}$ & $\begin{array}{l}\text { W5D003, T1WHZ1, T1WIJ6, T1WHQ5, T1WHU0, } \\
\text { C3VWV0, C3VWV3, C3VWZ7, Q5UHH6, } \\
\text { C3VWZ6 }\end{array}$ & No & $\begin{array}{l}\text { Triticum aestivum, Kengyilia } \\
\text { batalinii, Agropyron cristatum, } \\
\text { Agropyron mongolicum, } \\
\text { Eremopyrum bonaepartis, Triticum } \\
\text { dicoccoides }\end{array}$ \\
\hline \multirow[t]{4}{*}{ Q41540 } & IEMPGPPYLAK & $\begin{array}{l}\text { Alpha-amylase/trypsin } \\
\text { inhibitor CM16 }\end{array}$ & Q41540, M8B9L0 & Yes & $\begin{array}{l}\text { Triticum aestivum, Aegilops } \\
\text { tauschii }\end{array}$ \\
\hline & NYVEEQACR & $\begin{array}{l}\text { Alpha-amylase/trypsin } \\
\text { inhibitor CM16 }\end{array}$ & Q41540, M8B9L0 & Yes & $\begin{array}{l}\text { Triticum aestivum, Aegilops } \\
\text { tauschii }\end{array}$ \\
\hline & QECCEQLANIPQQCR & $\begin{array}{l}\text { Alpha-amylase/trypsin } \\
\text { inhibitor CM16 }\end{array}$ & Q41540, M8B9L0 & Yes & $\begin{array}{l}\text { Triticum aestivum, Aegilops } \\
\text { tauschii }\end{array}$ \\
\hline & SRPDQSGLMELPGCPR & $\begin{array}{l}\text { Alpha-amylase/trypsin } \\
\text { inhibitor } \mathrm{CMb}\end{array}$ & $\begin{array}{l}\text { P32936, P16159, A0A287PVL8, A0A1D5XLP1, } \\
\text { A0A1D5Y0V2, B5B0D5, Q41540, M8B9L0, } \\
\text { B9VRI3, Q45FA6 }\end{array}$ & No & $\begin{array}{l}\text { Secale cereale, Triticum macha, } \\
\text { Aegilops tauschii, Triticum } \\
\text { aestivum, Hordeum vulgare }\end{array}$ \\
\hline \multirow[t]{3}{*}{ P16851 } & $\begin{array}{l}\text { "EYVAQQTCGVGIVGSPVST } \\
\text { EPGNTPR }\end{array}$ & $\begin{array}{l}\text { Alpha-amylase/trypsin } \\
\text { inhibitor CM2 }\end{array}$ & P16851, D2TGC2, A0A1D6CFU7 & Yes & Triticum aestivum \\
\hline & ${ }^{*}$ TSDPNSGVLK & $\begin{array}{l}\text { Alpha-amylase/trypsin } \\
\text { inhibitor CM2 }\end{array}$ & P16851, A0A1D6CFU7, D2TGC2 & Yes & Triticum aestivum \\
\hline & $\begin{array}{l}\text { VLVTPGHCNVMTVHNTPYC } \\
\text { LGLDI }\end{array}$ & $\begin{array}{l}\text { Alpha-amylase/trypsin } \\
\text { inhibitor CM2 }\end{array}$ & P16851, D2TGC2 & Yes & Triticum aestivum \\
\hline \multirow[t]{4}{*}{ B9VRI3 } & "DYVEQQACR & $\begin{array}{l}\text { Alpha-amylase/trypsin } \\
\text { inhibitor CM16 }\end{array}$ & P32936, P16159, A0A287PVL8, B5B0D5, B9VRI3 & No & $\begin{array}{l}\text { Hordeum vulgare, Triticum } \\
\text { aestivum, Triticum macha }\end{array}$ \\
\hline & ${ }^{*}$ EVQMDFVR & $\begin{array}{l}\text { Alpha-amylase/trypsin } \\
\text { inhibitor CM16 }\end{array}$ & P32936, P16159, A0A287PVL8, B5B0D5, B9VRI3 & No & $\begin{array}{l}\text { Hordeum vulgare, Triticum } \\
\text { aestivum, Triticum macha }\end{array}$ \\
\hline & IETPGSPYLAK & $\begin{array}{l}\text { Alpha-amylase/trypsin } \\
\text { inhibitor CM16 }\end{array}$ & P16159, B5B0D5, B9VRI3 & Yes & Triticum aestivum, Triticum macha \\
\hline & QQCCGELANIPQQCR & $\begin{array}{l}\text { Alpha-amylase/trypsin } \\
\text { inhibitor CM16 }\end{array}$ & $\begin{array}{l}\text { P32936, P16159, A0A287PVL8, B5B0D5, B9VRI3, } \\
\text { Q45FA6 }\end{array}$ & No & $\begin{array}{l}\text { Hordeum vulgare, Triticum } \\
\text { aestivum, Triticum macha, Secale } \\
\text { cereale }\end{array}$ \\
\hline \multirow[t]{2}{*}{ C3VWC3 } & "DCCQQLADISEWCR & $\begin{array}{l}\text { Dimeric alpha-amylase } \\
\text { inhibitor }\end{array}$ & $\begin{array}{l}\text { A0A1D5VPJ2, A0A077RSX3, A0A077RQ83, } \\
\text { T1WHZ1, T1WIJ6, T1WHQ5, T1WHU0, C3VWZ7, } \\
\text { C3VWZ6, C3VWC3, T1WH90 }\end{array}$ & No & $\begin{array}{l}\text { Triticum aestivum, Kengyilia } \\
\text { batalinii, Agropyron cristatum, } \\
\text { Agropyron desertorum, Triticum } \\
\text { dicoccoides, Henrardia persica }\end{array}$ \\
\hline & "EHGVSEGQAGTGAFPSCR & $\begin{array}{l}\text { Alpha-amylase } \\
\text { inhibitor } 0.53\end{array}$ & $\begin{array}{l}\text { P01084, A0A077RSX3, C3VWL1, C3VWC7, } \\
\text { C3VWE8, C3VWL3, C3VW70, C3VWD7, } \\
\text { C3VWB8, C3VW96, C3VWC3, C3VWE6 }\end{array}$ & No & $\begin{array}{l}\text { Triticum aestivum, Kengyilia } \\
\text { batalinii, Agropyron cristatum, } \\
\text { Agropyron desertorum, Triticum } \\
\text { dicoccoides, Henrardia persica } \\
\end{array}$ \\
\hline N1QTW5 & ELAAISSNCR & Trypsin inhibitor CMc & P83207, A0A1D6D575, N1QTW5 & Yes & Triticum aestivum \\
\hline
\end{tabular}




\begin{tabular}{|c|c|c|c|c|c|}
\hline M8BV45 & "LYCCQELAEIPQQCR & $\begin{array}{l}\text { Alpha-amylase/trypsin } \\
\text { inhibitor CM3 }\end{array}$ & P11643, M8BV45, M0Y227, A0A1D5Y0V2 & No & $\begin{array}{l}\text { Hordeum vulgare, Aegilops } \\
\text { tauschii, Triticum aestivum }\end{array}$ \\
\hline & ${ }^{*}$ TDLLPHCR & $\begin{array}{l}\text { Alpha-amylase/trypsin } \\
\text { inhibitor CM3 }\end{array}$ & M8BV45, A0A1D5Y0V2 & Yes & $\begin{array}{l}\text { Aegilops tauschii, Triticum } \\
\text { aestivum }\end{array}$ \\
\hline \multirow[t]{2}{*}{ P93602 } & AEEGHVVEGR & $\begin{array}{l}\text { PUP88 protein; } \\
\text { member of trypsin/a- } \\
\text { amylase inhibitors } \\
\text { family from cereals }\end{array}$ & A0A1D6B7P2, P93602, M8CNM0, A0A1D6AS82 & Yes & $\begin{array}{l}\text { Triticum aestivum, Aegilops } \\
\text { tauschii }\end{array}$ \\
\hline & ELAVVPDYCR & $\begin{array}{l}\text { PUP88 protein; } \\
\text { member of trypsin/a- } \\
\text { amylase inhibitors } \\
\text { family from cereals }\end{array}$ & A0A1D6B7P2, P93602, M8CNM0 & Yes & $\begin{array}{l}\text { Triticum aestivum, Aegilops } \\
\text { tauschii }\end{array}$ \\
\hline \multirow[t]{4}{*}{ M8A1S2 } & "EFIAGIVGR & $\begin{array}{l}\text { Trypsin/alpha-amylase } \\
\text { inhibitor CMX2 }\end{array}$ & $\begin{array}{l}\text { Q43691, Q43723, M8A1S2, M8BAK8, M8B2U4, } \\
\text { A0A1D5XMK2, A0A1D6RXD1 }\end{array}$ & Yes & $\begin{array}{l}\text { Triticum aestivum, Triticum Urartu, } \\
\text { Aegilops tauschii }\end{array}$ \\
\hline & ${ }^{*}$ EITYESLNACR & $\begin{array}{l}\text { Trypsin/alpha-amylase } \\
\text { inhibitor CMX1/CMX3 }\end{array}$ & M8A1S2 & Yes & Triticum aestivum \\
\hline & QTCGYYLSAER & $\begin{array}{l}\text { Trypsin/alpha-amylase } \\
\text { inhibitor CMX1/CMX3 }\end{array}$ & $\begin{array}{l}\text { Q43691, Q43723, M8A1S2, M8BAK8, M8B2U4, } \\
\text { A0A1D5XMK2, A0A1D6RXD1 }\end{array}$ & Yes & $\begin{array}{l}\text { Triticum aestivum, Triticum Urartu, } \\
\text { Aegilops tauschii }\end{array}$ \\
\hline & EECNLETVFGR & $\begin{array}{l}\text { Trypsin/alpha-amylase } \\
\text { inhibitor CMX1/CMX3 }\end{array}$ & M8A1S2, M8B2U4, A0A1D5XMK2 & Yes & $\begin{array}{l}\text { Triticum aestivum, Triticum Urartu, } \\
\text { Aegilops tauschii }\end{array}$ \\
\hline A4GG11 & LPNVVDASGDGAYVCK & $\begin{array}{l}\text { Dimeric alpha-amylase } \\
\text { inhibitor }\end{array}$ & A4GG11 & Yes & Aegilops longissima \\
\hline C4P5B7 & DCCQQLADINDEWCR & $\begin{array}{l}\text { Monomeric alpha- } \\
\text { amylase inhibitor }\end{array}$ & C4P5B7 & Yes & Triticum dicoccoides \\
\hline A0A1D5Y0V2 & YFMGPK & N/A & $\begin{array}{l}\text { Unable to match any Uniprot ID due to short } \\
\text { sequence. }\end{array}$ & N/A & N/A \\
\hline \multirow[t]{2}{*}{ A0A1D5UB33 } & ELEAVSEECR & $\begin{array}{l}\text { Uncharacterized } \\
\text { protein }\end{array}$ & A0A1D5UB33, A0A1D5TQ92, A0A1D5Y0V2 & Yes & Triticum aestivum \\
\hline & LEGVPEGCTR & $\begin{array}{l}\text { Uncharacterized } \\
\text { protein }\end{array}$ & A0A1D5UB33, M7YQH9, A0A1D5Y0V2 & Yes & Triticum aestivum, Triticum urartu \\
\hline Q6S5B1 & LPEWMTSASIYSPGKPYLAK & $\begin{array}{l}\text { Alpha-amylase/trypsin } \\
\text { inhibitor CM3 }\end{array}$ & $\begin{array}{l}\text { P17314, Q53YX8, Q6S5B1, A0A1D5XLP1, } \\
\text { A0A1S6KXP9 }\end{array}$ & Yes & $\begin{array}{l}\text { Triticum aestivum, Triticum } \\
\text { turgidum subsp. Durum }\end{array}$ \\
\hline
\end{tabular}

